\title{
Neue Befunde zu den sedimentologisch-stratigraphischen Lagerungsverhältnissen und zur Alterszuordnung der äolischen Decksedimente auf dem Niederrheinischen Höhenzug
}

\author{
Helmut Siebertz *)
}

\author{
Weichselian, aeolian sediments, periglacial environment, sedimentology, stratigraphie, solifluction \\ North-West Germany, Lower Rhine embayment
}

\begin{abstract}
Kurzfassung: Auf dem Niederrheinischen Höhenzug lassen sich im Jung-Weichsel-Glazial (oberes Pleniglazial) nach der Steinsohlenbildung (ca. 28.000 a BP) mehrere Sedimentgenerationen sedimentologisch-stratigraphisch belegen. Diese sind: 1. Lösse und Varietäten, 2. Flug- und Treibsande.
\end{abstract}

Bei den Lössen lassen sich der älteste Löß (lokal in Resten vorzufinden), der jüngere Löß sowie der jüngste Löß gliedern. Die jüngere und jüngste Lößablagerung ist durch einen Solifluktionshorizont unterbrochen, der als Äquivalent einer Klimaschwankung mit Bodenbildung gesehen wird. Ihm wird ein relatives Alter von 20.000 a BP zugesprochen. Der Solifluktion folgt der typische ungeschichtete jüngste Weichsellöß, dessen Ablagerung in der ältesten Dryaszeit endet und damit einen Ablagerungszeitraum von $8.000 \mathrm{a}$ umfaßt (Tab. 1).

Für das Jung-Weichsel-Glazial lassen sich die Flugsande auch durch eine ältere (untere) sowie ältere (obere) Sedimentation belegen. Beide Flugsandablagerungen sind lokal durch Fließerdehorizonte unterbrochen, wobei der oberste Fließerdeabschnitt etwa zeitlich mit der großen Abtragung um etwa 20.000 a BP zusammenfallen dürfte. Der ältere (untere) Flugsand zeigt mit den in der Saigerungsabfolge liegenden Sedimenten eine flächenhafte Ausdehnung (Kt. i. d. Anl.); die sedimentologisch-stratigraphischen Lagerungsverhältnisse ermöglichen es, ihn zwischen dem ältesten sowie dem jüngeren Löß zeitlich einzuordnen. Damit hat der ältere (untere) Flugsand ein relativ höheres Alter als die jüngere Lößablagerung (Tab. 1).

Für das Spätglazial läßt sich ferner ein jüngerer (unterer) sowie ein jüngerer (oberer) Treib- bzw. Flugsand nachweisen, wobei die Hauptsedimentation des Treibsandes vornehmlich in der jüngeren Dryaszeit stattfand (Tab. 1).

Die Decksedimente liegen im Grenzbereich der nördlichen Lößgrenze. Aufgrund der vorliegenden Befunde lassen sie sich nun in die niederrheinische Löß- sowie in die norddeut-

*) Anschrift des Autors: Dipl.-Geogr. Dr. H. SIEBERTZ, Alte Schulstr. 16, D-5300 Bonn. sche und niederländisch-belgische Flugsandstratigraphie des Weichsel-Glazials sinnvoll einordnen.

[New results of the sedimentological-stratigraphical bedding conditions and age dating of the aeolian cover sediments on the Lower Rhenish ridge (Lower Rhine area)

Abstract: On the Lower Rhenish ridge several cover sediments of the younger Weichsel glacial period are bedded by a stoneline aged circa 28.000 a BP. The stratigraphically above the stoneline can be described by different generations of cover sediments (oldest loess, older [lower and upper] flying sand, younger and youngest loess, younger [lower and upper] drift sand).

The oldest loess is only to find in some small local spots. The younger and youngest loess sedimentation is interrupted by solifluction; the solifluction is comparable to a climatal fluctuation. Above this horizon lies the typical unstratified youngest loess (upper Pleniglacial). It was deposited from circa 20.000 a BP up to the oldest Dryas period at 12.000 a BP. This deposition of the youngest loess sedimentation comprised an intervall of circa 8.000 years (table index No. 1).

The stratigraphically of the older (lower and upper) flying sand is comparable to the stratigraphically of the loess sedimentation. The flying sands are interrupted by several solifluctions, too. The sequence of the sedimentation of the older (lower) flying sand concludes, that the sediment can be relatively aged between the oldest loess and the younger loess sedimentation. The younger (lower and upper) drift sand is primary aged of the younger Dryas period (table index No. 1).

These aeolian sediments are deposited in the nearness of the Northern Loess Boundary in the western part of Germany. The new results comply with the stratigraphical loess nomenclature of the Lower Rhine district and of the flying sand nomenclature (Weichsel glacial period) beyond the loess boundary in the North-West landscape region (Belgium, Netherland, North-Germany). 


\section{Problemstellung}

Die äolischen Decksedimente auf dem Niederrheinischen Höhenzug sind seit den fünfziger Jahren Gegenstand intensiver Untersuchungen (vgl. SIEBERTZ 1992). Es steht heute außer Zweifel, daß die ursprünglich als Sandlöß angesprochenen Sedimente nur eine untergeordnete Rolle spielen und die Hauptablagerung aus Löß besteht (s. Kt. in der Anl.). Dieser neue sedimentologische Befund wird durch die Bodenkartierung von PAAS (1985) bestätigt.

Aufgrund der Untersuchungen von SIEBERTZ (1983) schienen die Lagerungsverhältnisse der Decksedimente bekannt zu sein. Es ließen sich eine ältere Flugsandablagerung sowie eine jüngere Lößakkumulation sedimentologisch-stratigraphisch und räumlich voneinander trennen. Der nordwestliche Raum des Reichswaldes blieb jedoch weitgehend unberücksichtigt. Die Untersuchung dieser Restfläche führte nun auch zu intensiveren Aufgrabungen der Decksedimente auf der Uedemer Hochfläche. Daraus folgte eine neue Be- wertung und Typisierung der äolischen Sedimente auf dem Höhenzug (vgl. SIEBERTZ 1990).

Die neuen Befunde zeigen nun eine größere sedimentologische Vielfalt einerseits, andererseits verlangen aber die Sedimente auch eine differenziertere stratigraphische Ansprache (Abb. 1; Karte). Aufgrund der Befunde ist eine neue chronostratigraphische Alterszuordnung der Ablagerungen in die niederrheinische Löß- und die norddeutsche (sowie belgisch-niederländische) Flugsandstratigraphie der Weichsel-Kaltzeit verbunden.

\section{Die stratigraphische Lagerung der Decksedimente nach neuesten Befunden}

Die Basis der Decksedimente bildet in den meisten Fällen die Steinsohle oder der saalezeitliche Sander. Dadurch lassen sich die Windsedimente recht gut vom Untergrund abgrenzen. Die Steinsohle bildet eine wichtige stratigraphische Marke sowie einen

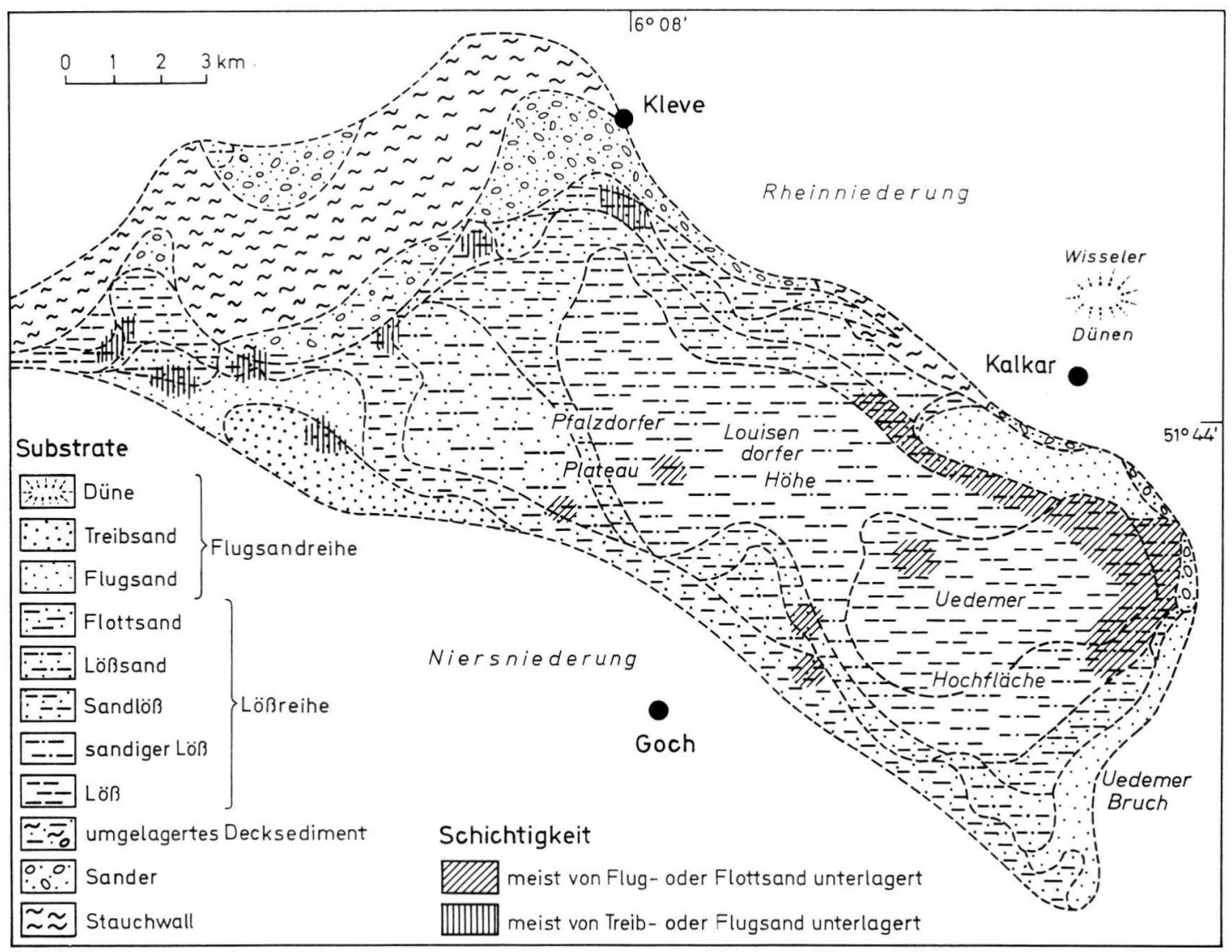

Abb. 1: Äolisches Decksedimentverteilungsbild vom nördlichen Niederrheinischen Höhenzug (HERBST 1988). 


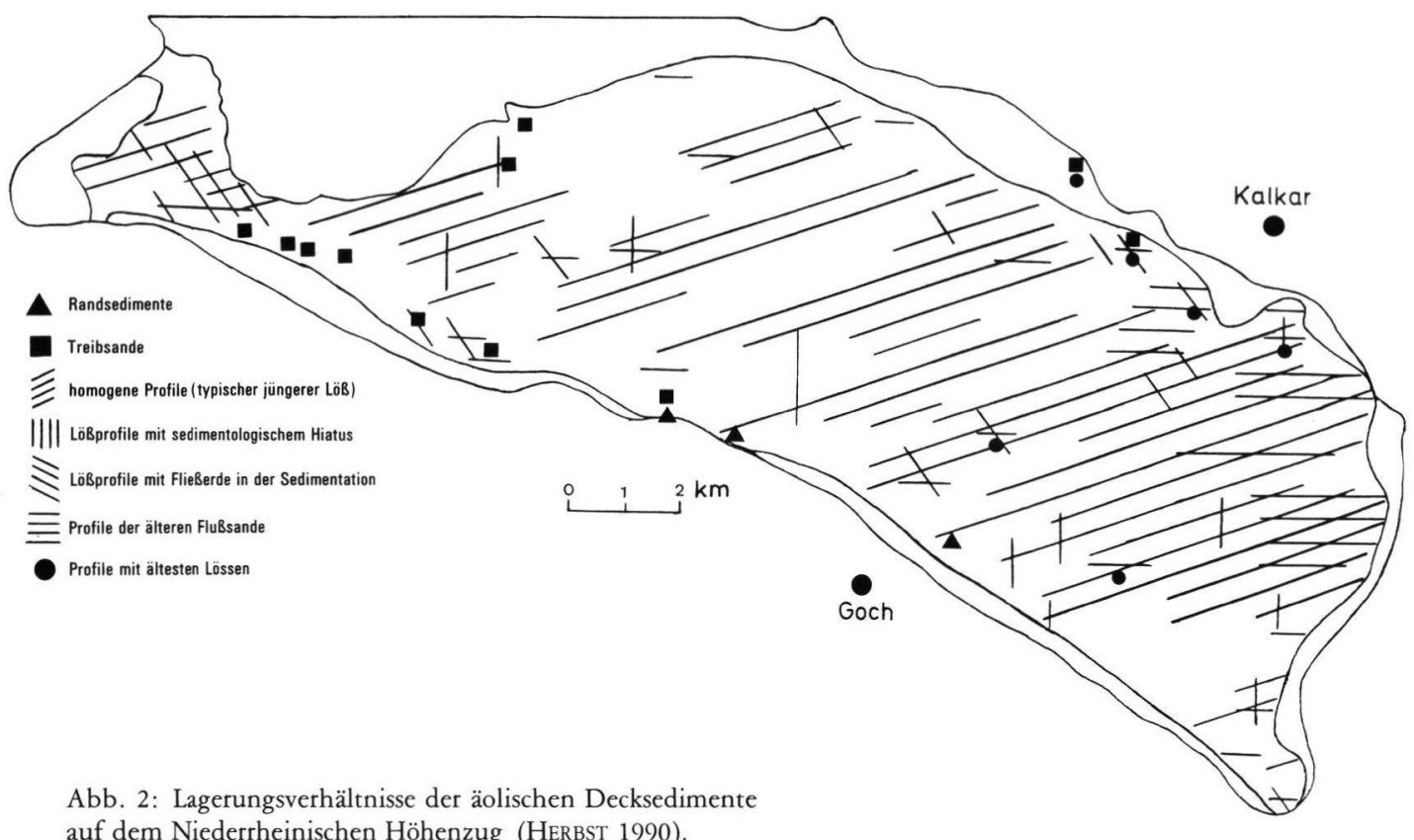

auf dem Niederrheinischen Höhenzug (HERBST 1990).

deutlichen sedimentologischen Hiatus zwischen dem Liegenden und dem Hangenden (vgl. SieBERTZ 1987). Oberhalb dieser Steinsohle lassen sich nun sedimentologisch-stratigraphisch folgende Decksedimente gliedern: 1) ältester Löß: er befindet sich nur im Randbereich der Hochfläche in geschützten Lagen, wo er die Erosion überdauern konnte. Die Mächtigkeit beträgt im allgemeinen $1 \mathrm{dm}$ und weniger; das Vorkommen ist auf die Basis weniger Profilaufgrabungen beschränkt, so daß ein flächenhafter Nachweis bisher nicht möglich ist (Abb. 2). Stratigraphisch wird er entweder von den älteren Flugsanden oder von den jüngeren Lößsedimenten überlagert (Tab. 1). Der älteste Löß hat durchweg eine sehr feine Korngrößenzusammensetzung, die der des sandigen Lösses und Lösses entspricht (vgl. Karte).

2) Älterer (unterer und oberer) Flugsand: die Flugsandablagerungen sind aufgrund ihrer relativ grobkörnigen Zusammensetzung vornehmlich auf die Randbereiche der Hochfläche beschränkt (Abb. 2). Das Zentrum des Höhenzuges (Abb. 1) bleibt deshalb von der Flugsandsedimentation ausgenommen. Lediglich im Osten des Höhenzuges läßt sich eine zusammenhängende (ältere untere) Flugsandablagerung nachweisen. Diese besteht jedoch nur am Ostrand aus Flugsanden; sie wird zum Zentrum der Uedemer Hochfläche hin aufgrund der windbedingten Saigerungsabfolge von feineren Sedimenten abgelöst (vgl. Karte), wobei die Mächtigkeit schnell bis auf wenige Dezimeter abnimmt (Abb. 15 in SIEBERTZ 1992).
Stratigraphisch liegen die älteren (unteren) Flugsande unter der jüngeren Lößablagerung (Tab. 1). Die der Fließerde folgende ältere (obere) Flugsand ist aufgrund der geringen Mächtigkeit und Streuweite nur auf die Randbereiche beschränkt. Beim Fehlen des Fließerdehorizontes ist eine Gliederung in eine untere und obere Flugsandablagerung bisher nicht möglich, so daß in Abbildung 2 bei der Sedimentansprache nur der , untere' Flugsand stellvertretend für beide Sedimente in Erscheinung tritt.

3) Jüngerer sowie jüngster Löß und Äquivalente: der gesamte Höhenzug ist vorzugsweise von dieser Sedimentgruppe bedeckt (Karte). Nach neuesten Erkenntnissen gehören diese Sedimente allerdings keiner einheitlichen Ablagerungsperiode an; es lassen sich zwei Ablagerungsphasen mit Hilfe eines Solifluktionshorizontes gliedern (in Abb. 2 sind beide Ablagerungen als typischer jüngerer Löß zusammengefaßt). Zu ihnen gehört der jüngere Löß, der nicht flächenhaft nachgewiesen werden kann und nur in Einzelprofilen am Rande der Hochfläche lagert (Abb. 2). Stratigraphisch liegt er zwischen den älteren (unteren) Flugsanden und dem jüngsten Löß (Tab. 1).

Der jüngere Löß steht nicht unmittelbar an der Oberfläche an, sondern er wird immer vom jüngsten Löß überlagert. Zwei Merkmale ermöglichen es, beide Sedimentkomplexe zu trennen: dies sind a) das Vorkommen von einem sedimentologischen Hiatus im Profilaufbau (Aufgrabungen 23, 28, 54, 71 in Tab. 2), oder b) das Auftreten eines Solifluktionsabschnittes im Profil (Aufgrabungen 89, 106, 109, 163, 
Tab. 1: Chronostratigraphie der äolischen Decksedimentbildung auf dem Niederrheinischen Höhenzug (HERBST 1990).

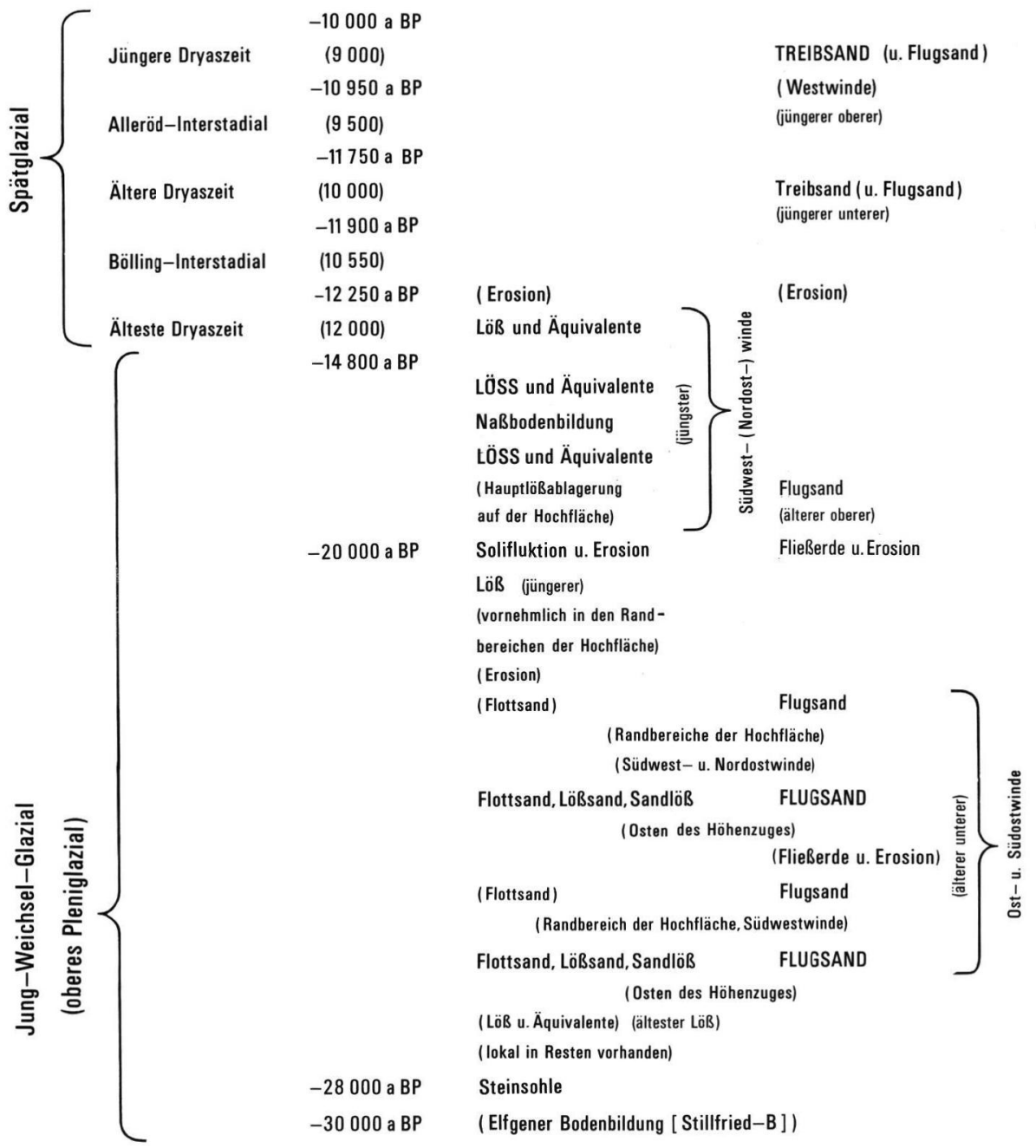

166, 174 in Tab. 2). Ist der Solifluktionshorizont erodiert, so läßt sich zwischen dem jüngeren und jüngsten Löß ein sedimentologischer Hiatus nachweisen, der sich im Hangenden des Profils durch eine deutliche Vergröberung der Sedimente auswirkt (Tab. 2). Die Solifluktion ist in den meisten Fällen durch einen Verbraunungs- oder Oxidationshorizont sowie durch das Vorhandensein kleinerer Kiese in den mittleren Profilabschnitten gekennzeichnet. Selbst bei der Aufgrabung im Gelände läßt sich die Solifluktion nicht in situ nachweisen, sondern nur durch eine entsprechende sedimentologische Untersuchung im Labor.

Profile, in denen die Solifluktion die beiden Lößablagerungen gliedert, beginnen im Liegenden mit einer zum Zentrum hin zunehmenden Vergröberung der Sedimente bis zum Solifluktionsabschnitt, die dann zum Hangenden aber in der Körnung wieder feiner wird (Tab. 2). Im Konzentrationsdreieck (Abb. 3) sind die Solifluktionsabschnitte verschiedener Profile zusammengestellt; sie zeigen eine relativ einheitliche Zusammensetzung. Das Grobkorn $(2-1 \mathrm{~mm})$ ist in nur ganz wenigen Fällen mit bis zu $2 \%$ Kornanteil beteiligt; die meisten Sedimentabschnitte enthalten keinen Grobsand.

Der Solifluktion folgt im Hangenden der jüngste (typische) Löß (Tab. 1); dieser bildet die Hauptlößablagerung auf der Hochfläche mit den unmittelbar an der Oberfläche anstehenden Sedimenten. Diese Ablagerungen zeigen sedimentologisch einen homogenen Profilaufbau (vgl. SIEBERTZ 1983, 1990). Im Zentrum der Hochfläche, wo der jüngere Löß an der Basis fehlt, wird er unmittelbar von der Steinsohle unterlagert. $\mathrm{Zu}$ dieser Decksedimentablagerung gehören auch die Randsedimente. Es sind meistens Flottsandprofile im Südwesten des Höhenzuges, die lokal von Flugsandstreifen durchsetzt sind (Abb. 2).

4) Jüngerer (unterer und oberer) Treib- und Flugsand: der Treibsand als jüngstes Decksediment tritt 
Tab. 2: Profile mit sedimentologischem Hiatus (Feinheitsgrade unterstrichen) und Solifluktion (Feinheitsgrade fettgedruckt) mit Profillokalitäten. In beiden Fällen bildet das Liegende den jüngeren Löß, das Hangende den jüngsten, typischen, ungeschichteten Weichsel-Löß.

\begin{tabular}{|c|c|c|c|c|c|c|c|c|c|c|c|}
\hline \multicolumn{2}{|c|}{ Aufgrabung } & 23 & 28 & 54 & 71 & 89 & 106 & 109 & 163 & 166 & 174 \\
\hline \multicolumn{2}{|l|}{ TK 25} & 4202 & 4303 & 4303 & 4303 & 4203 & 4202 & 4202 & 4203 & 4203 & 4203 \\
\hline \multicolumn{2}{|l|}{$\mathrm{R}$} & 11000 & 15220 & 18960 & 19950 & 14750 & 99550 & 98700 & 17670 & 18350 & 11640 \\
\hline \multicolumn{2}{|l|}{$\mathrm{H}$} & 30660 & 27720 & 28700 & 27150 & 30180 & 35080 & 35500 & 31050 & 31510 & 35720 \\
\hline \multirow{2}{*}{. } & $\iota$ & & & & & 67,2 & & & & 70,2 & \\
\hline & $\vartheta$ & & & & & 69,6 & & 69,2 & & 73,2 & \\
\hline \multirow{7}{*}{ 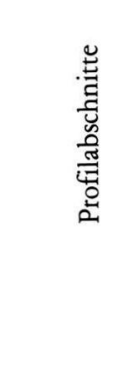 } & $\eta$ & & & & & 71,4 & & 68,6 & 70,1 & 74,5 & 60,0 \\
\hline & $\varrho$ & 59,8 & & 63,3 & & 65,8 & 67,8 & 67,5 & 72,4 & 72,8 & 61,6 \\
\hline & $\varepsilon$ & 59,3 & & 65,0 & & 50,1 & 68,4 & 45,6 & 69,2 & 67,8 & 56,6 \\
\hline & $\delta$ & 65,2 & 64,9 & 70,8 & 60,9 & 62,9 & 53,8 & 49,7 & 47,6 & 51,5 & 48,5 \\
\hline & $\gamma$ & 70,4 & 65,8 & 72,1 & 62,3 & 64,0 & 52,1 & 65,0 & 61,9 & 62,6 & 55,6 \\
\hline & $\beta$ & 73,7 & 71,8 & 71,8 & 73,4 & 59,6 & 69,6 & 69,8 & 65,8 & 69,5 & 63,6 \\
\hline & $\alpha$ & 69,3 & 71,1 & 69,3 & 70,9 & 69,8 & 71,3 & 69,7 & 70,3 & 66,6 & 67,2 \\
\hline \multicolumn{2}{|l|}{ Basis } & St & $\mathrm{Sa}$ & St & St & St & St & St & St & St & St \\
\hline
\end{tabular}

$\mathrm{Sa}=$ Sander

St $=$ Steinsohle

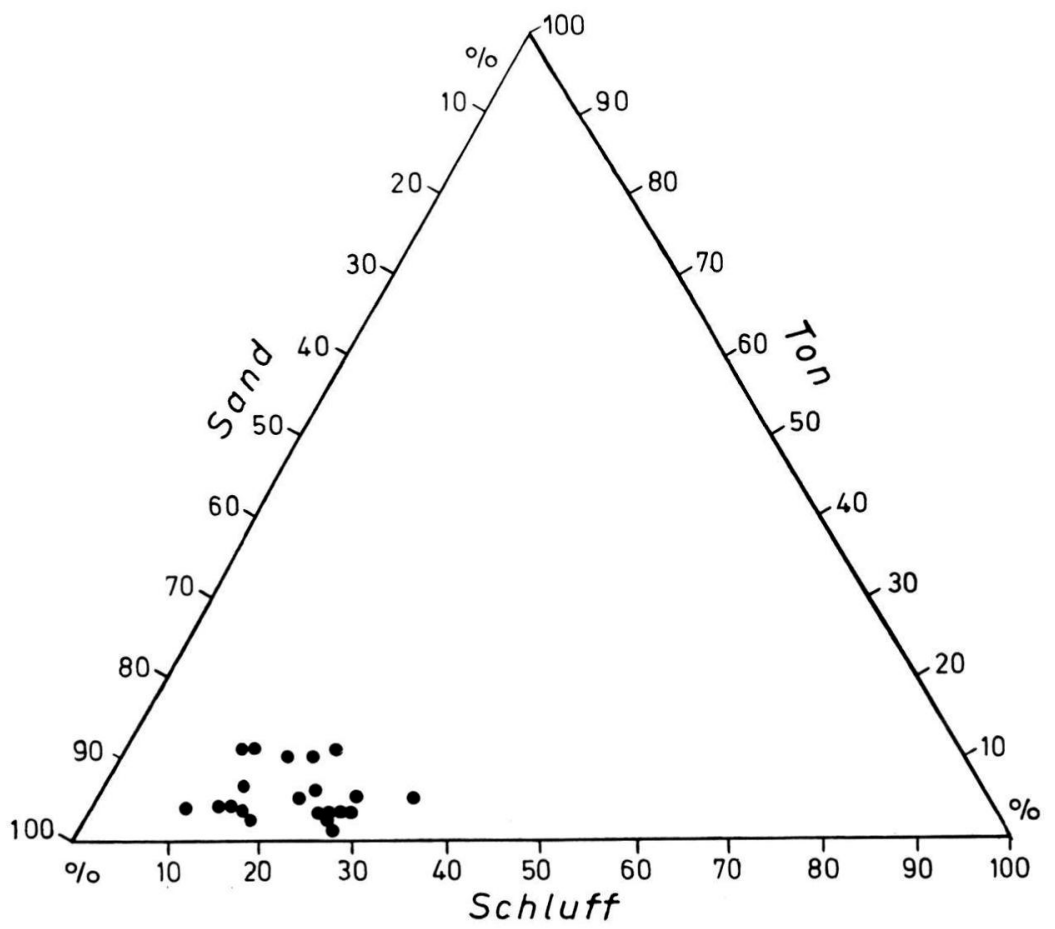

Abb. 3:

Korngrößenzusammensetzung der Solifluktionsabschnitte in den Lößprofilen. 
hier als sedimentologisches Äquivalent zum spätglazialen Dünensand in der Rhein- und Maasniederung auf (Tab. 1). Der Treibsand bildet die gröbste Ablagerung auf dem Höhenzug (Karte). In seiner flächenhaften Ausdehnung ist er hauptsächlich auf den Nordwestrand des Höhenzuges beschränkt (Abb. 2). In der Regel folgt ihm - außer dem Flugsand kein feineres Sediment in der Saigerungsabfolge (vgl. Kap. 8 in SIEBERTZ 1992). Im Nordwesten liegt der Treibsand über den älteren Flugsanden bzw. im Osten des Höhenzuges über den jüngsten Lößsedimenten (Flottsanden; Karte).

\section{Die Altersgliederung der äolischen Decksedimente nach neueren Erkenntnissen}

Nach dem derzeitigen Kenntnisstand ist eine absolute Altersdatierung der äolischen Decksedimente mit Hilfe paläopedologischer Untersuchungsmethoden nicht möglich. Die komplizierten Lagerungsverhältnisse (Tab. 1) allerdings lassen erahnen, daß eine relative Alterszuordnung schwierig durchzuführen ist. Hier sind die genaueren Kenntnisse der Sedimentstratigraphie sehr hilfreich. Sie ermöglichen es, die Decksedimente nun der niederrheinischen sowie norddeutschen und niederländisch-belgischen Sediment- und Bodenchronostratigraphie sinnvoll zuzuordnen.

Die Windsedimente auf dem Niederrheinischen Höhenzug sind alle jungweichselzeitlich und jünger (Tab. 1). Nach PAAS (1968) wird im Niederrheingebiet der ältere und jüngere Weichsellöß durch den Elfgener Boden getrennt, der in der mitteleuropäischen Bodenstratigraphie mit dem Stillfried B gleichzusetzen ist. Dieser fossile Boden (ca. 30.000 a BP) ist auf dem Höhenzug (bisher) nicht nachweisbar. Dafür wird die Basis durchweg von einer Steinsohle gebildet, die von SIEBERTZ (1987) als Indikator für feuchtere Klimabedingungen gedeutet wurde und genetisch als Äquivalent einer Fließerde oder Bodenbildung betrachtet werden kann. Sie wird im Alter etwas jünger als der Elfgener Boden angesehen, so daß der Steinsohle in ihrer jetzigen Lage bei der Plombierung durch die Decksedimente ein Alter von circa 28.000 a BP zugesprochen werden darf (Tab. 1).

Eine wichtige Marke für die Sedimentstratigraphie bildet der Solifluktionshorizont zwischen dem jüngeren und dem jüngsten Weichsel-Löß bzw. der Fließerdeabschnitt zwischen dem älteren unteren und älteren oberen Flugsand. Beide sind vergleichbar der Steinsohle mit Sandeinlagerungen von PAAS (1968), der ein Alter von etwa 20.000 a BP zugewiesen wurde. Der Solifluktion folgt die eigentliche kontinuierliche, ungeschichtete, typische homogene Lößablagerung auf der Hochfläche, deren Sedimentation bis in die älteste Dryaszeit (ca. 12.000 a BP) anhielt und damit einen Ablagerungszeitraum von etwa $8.000 \mathrm{a}$ umfaßt (Tab. 1).

Die jüngeren Treib- und Flugsande wurden ausnahmslos im Spätglazial abgelagert; sie sind jünger als die älteste Dryaszeit, zumal auf dem Höhenzug bis ins Spätglazial hinein der Löß zur Ablagerung kam (Tab. 1). Sie lassen sich in eine untere und obere Treibsandablagerung gliedern, die in diversen Profilen durch einen kräftigen $\mathrm{B}_{\mathrm{s}}$-Horizont voneinander getrennt sind. Aufgrund der meist mächtigen Sedimentationsrate nach der Podsolierung läßt sich die Hauptablagerung mit der jüngsten Dryaszeit in Verbindung bringen.

\section{Diskussion zur chronostratigraphischen Einordnung der weichselzeitlichen Decksedimente vom Niederrheinischen Höhenzug im Vergleich zu den Nachbargebieten}

Die Decksedimentablagerungen auf dem Niederrheinischen Höhenzug liegen im Grenzbereich der klassischen nördlichen Lößgrenze. Zu dieser Problematik hat SiEBERTZ (1988) ausführlich Stellung genommen. Südlich dieser Grenze befindet sich das klassische Lößgebiet, nördlich davon die großen zusammenhängenden Areale der Flugsandablagerungen (Belgien, Niederlande, Norddeutschland). Flugsande aus der Weichsel-Kaltzeit sind deshalb in der niederrheinischen Lößstratigraphie nicht zu finden und auch völlig unüblich.

Daß nicht nur der Löß, sondern auch Flugsande in der Weichsel-Kaltzeit verfrachtet und abgelagert wurden, zeigen diverse Untersuchungen im norddeutschen Tiefland und angrenzenden Gebieten. Die Untersuchungen zeigen, daß sich für das obere Pleniglazial zwei Flugsandablagerungen nachweisen lassen, die durch eine Steinsohle voneinander getrennt sind (Abb. 6 in SieberTZ 1987). Von MAARLEVELd (1976) werden für das Weichsel-Hochglazial der ältere untere Flugdecksand I sowie der ältere obere Flugdecksand II unterschieden; beide Sedimentablagerungen werden durch den Beuningen-Boden bzw. durch Kiesablagerungen als Äquivalent getrennt. Dies deutet zumindest auf eine Ruhephase der Sedimentation unter feuchtem und damit verbunden wärmerem Klima hin.

In diesen Zeitraum des Hochglazials fallen auch die älteren Flugsandablagerungen vom Niederrheinischen Höhenzug. Sie können im Alter mit den älteren unteren und oberen Flugsanden von MAARLEVELD (1976) verglichen werden. Die in verschiedenen Flugsandprofilen im Osten, aber auch im Süd- und Nordwesten des Höhenzuges auftretenden Fließerden 
zeigen, daß während der Flugsandablagerungen feuchtere Klimaperioden die Sedimentationen unterbrachen und örtlich zur Bildung von Oxidationshorizonten führten. Sieht man die Fließerden sowie die Oxidationszonen als Äquivalente einer Bodenbildung an, so lassen sich zumindest zwei Flugsandablagerungen gliedern, die allerdings aufgrund der meist geringen Mächtigkeit nach der Fließerdebildung des älteren (unteren) Flugsandes genetisch nicht den gesamten Zeitraum des oberen Pleniglazials bis zur älteren Dryaszeit auszufüllen vermögen (Tab. 1), wie dies in den Nachbargebieten der Fall ist.

Nicht in allen Flugsandprofilen läßt sich die Fließerde nachweisen. $\mathrm{Da}$ die ältere (untere) Flugsandbildung mit ihren Folgesedimenten in der Saigerungsabfolge flächenhaft abgelagert wurde und die Basis des jüngeren Lösses bildet (Karte), können die Flugsandprofile, die sich nicht durch einen $\mathrm{B}_{\mathrm{s}}$-Horizont gliedern lassen, der älteren (unteren) Ablagerungsperiode zugeschrieben werden.

Für den Niederrheinischen Höhenzug läßt sich mit Hilfe der Solifluktion nun auch eine Zweiteilung des jungweichselzeitlichen Lösses durchführen (Tab. 1), wie sie von PAAS (1968) für den niederrheinischen Raum erarbeitet wurde. Die ursprüngliche Ansicht über die mehr oder weniger deutliche sedimentologische Einheitlichkeit dieser Ablagerung von SIEBERTZ (1983) ist somit ergänzungsbedürftig.

Der Solifluktion um etwa 20.000 a BP wird in der Lößstratigraphie eine große Bedeutung beigemessen. Die von SEMMEL (1990) erwähnte bedeutsame letzte, besonders kräftige Erosionsphase für das frühe Jungwürm (Jungweichsel) läßt sich bei Hammen et al. (1967), PaAs (1968) sowie ZaGWIjN \& PAEPE (1968) durch Erosion oder Steinsohlenbildung nachweisen. Mit ihr scheint eine kräftige Erosion einherzugehen, die auch für das geringe Vorkommen von älterem Jungweichsellöß auf dem Niederrheinischen Höhenzug in Verbindung zu setzen ist.

Die stratigraphischen Lagerungsverhältnisse auf dem Höhenzug tragen dieser Problematik Rechnung. Sie zeigen, daß im Jung-Weichsel-Glazial mehrere Erosionsphasen die Sedimentablagerung unterbrachen bzw. abgelagerte Sedimente erodierten (Tab. 1). Der Solifluktionshorizont deutet auf eine sedimentäre Ruhephase hin, die unter feuchten Klimabedingungen unter Einwirkung fremder Sedimente initiiert wurde. Auf dem Höhenzug liegen die homogenen Profile (jüngster Löß) sowie die von Solifluktion unterbrochenen Profile (jüngerer und jüngster Löß) oft dicht beieinander (Abb. 2).

Die dryaszeitlichen Ablagerungen haben die Eigenschaft, daß sie östlich der Ausblasungsgebiete liegen. Darauf wies bereits BREDDIN $(1926,1927)$ hin, der erkannte, daß die spätglazialen Ablagerungen östlich der Niederterrasse liegen. Die Treibsande auf dem Höhenzug zeigen auch die für spätglaziale Ablagerungen typischen Sedimentationsbedingungen (Karte). Daß die Gleichaltrigkeit der Sediment- sequenz von Flugsand, Flottsand, Sandlöß und Löß zu relativieren ist, war dem Autor jedoch noch nicht bekannt.

Selbst MÜLlER $(1954,1959)$ war noch der Auffassung, daß Flugsand, Sandlöß, Löß und Niederterrasse gleichen Alters sind, obwohl dies schon von WILCKENS (1924) angezweifelt wurde. Der Autor bemerkt, daß die Flugsande mit dem Löß nichts zu tun haben; sie vertreten ihn weder, noch stehen sie mit seiner Bildung in ursächlichem Zusammenhange. Aus den stratigraphischen Lagerungsverhältnissen folgert WILCKENS (1924), daß die Flugsande jünger als die Niederterrasse und jünger als der Löß sind. Ihre Entstehung fällt in die zweite Hälfte der Dryasperiode (Ende der Yoldiazeit). Dies trifft zweifellos für die spätglazialen Treibsande des Höhenzuges zu (Karte; Tab. 1), zumal eine begrifflche Trennung zwischen Flugsand und Treibsand aufgrund der seinerzeit fehlenden sedimentologischen Kenntnisse noch nicht möglich war.

Dünensande sowie Treibsande sind verstärkt im Spätglazial abgelagert worden. Die meisten Autoren gliedern verschiedene Decksedimente (cover sands), welche den einzelnen Dryaszeiten zugeordnet werden. $\mathrm{Zu}$ ihnen gehören u. a. ARENS (1964), DÜCKer \& MAARLEVELD (1957), VAN DER Hammen et al. (1967), MAarieVeld (1976), Meyer (1981), VIERHUfF (1967) sowie ZAGWIJN \& PAPE (1968). PONS (1957) gliedert alt- sowie jungdryaszeitliche Windaufwehungen im Maasgebiet. Während sich das Bölling-Interstadial nicht in allen Untersuchungen nachweisen läßt, beschreibt ARENS (1964) als Äquivalent einen humosen Horizont, ZAGWIJN \& PAEPE (1968) Torf und Lehm bzw. in Belgien den Stabroek B-Boden. Das Alleröd-Interstadial ist jedoch in allen Untersuchungen durch einen humosen Horizont bzw. durch den Usselo-Boden in den Niederlanden belegt (vgl. Abb. 6 in SIEBERTZ 1987).

Das Fehlen fossiler Bodenhorizonte in den entsprechenden Sedimentaufgrabungen auf dem Niederrheinischen Höhenzug, aber auch das Vorkommen von lediglich nur einem $\mathrm{B}_{h}, \mathrm{~B}_{\mathrm{s}}$ oder $\mathrm{C}_{\mathrm{s}}$-Horizont im Profil, läßt nicht darauf schließen, daß die Anfänge der Treibsandbildung nicht bereits in der älteren Dryaszeit einsetzten. Für die Treib- und Flugsande kann mit Hilfe der $\mathrm{B}_{\mathrm{s}}$-Horizonte zumindest in diversen Profilen eine anfängliche altdryaszeitliche Sedimentation belegt werden.

Aufgrund dieser Befunde läßt sich, wie in den Nachbargebieten (Abb. 6 in SIEBERTZ 1987), ein jüngerer (unterer) und jüngerer (oberer) Treib- bzw. Flugsand gliedern, so daß auch hier die stratigraphische Lücke zu den spätglazialen Flugsandgebieten im norddeutschen und angrenzenden Raum geschlossen werden kann. Die teilweise recht große Sedimentmächtigkeit, die dem $B_{s}$-Horizont des jüngeren (unteren) Treibund Flugsandes im Hangenden folgt, läßt die Erkenntnis zu, daß die Hauptablagerung aber erst in 
der jüngeren Dryaszeit stattfand (Tab. 1). Damit fallen die Ablagerungen zeitlich mit den großen Dünensandablagerungen östlich der Maas und im Rheingebiet (Schlebusch-Wahner Heide bei Köln) zusammen, die schon von PoNs (1957) sowie JuX (1956) mit der jüngeren Dryaszeit in Verbindung gebracht wurden.

Für die weichselzeitlichen Decksedimentablagerungen auf dem Niederrheinischen Höhenzug gilt, daß sie polygenetisch abgelagert und zu dem heutigen Sedimentationsverteilungsbild zusammengeschweißt wurden (Karte).

\section{Schriftenverzeichnis}

ARens, H. (1964): Zur Altersdatierung der Flugsande am Westrand des Münsterschen Kreidebeckens. - Decheniana, 117: 133-140, 2 Abb.; Bonn.

BREDDIN, H. (1926): Löß, Flugsand und Niederterrasse am Niederrhein. - Jb. preuß. geol. L. A., 46: 635-662, 2 Fig.; Berlin.

- (1927): Löß, Flugsand und Niederterrasse im Niederrheingebiet, ein Beitrag zur Frage der Entstehung des Löß. - Geol. Rdschau, 18: 72-78, 1 Abb.; Stuttgart.

DÜCKer, A. \& MaArleveld, G. C. (1957): Hoch- und spätglaziale äolische Sande in Nordwestdeutschland und in den Niederlanden. - Geol. Jb., 73: 215-234, 8 Abb., 5 Tab.; Hannover.

Hammen, T. v. d., Maarleveld, G. C., Vogel, J. C. \& ZAGwijn, W. H. (1967): Stratigraphy, Climatic Succession and Radiocarbon Dating of the last Glacial in the Netherlands. - Geol. en Mijnb., 46: 79-95, 9 Fig.; s'Gravenhage.

JUX, U. (1956): Über Alter und Entstehung von Decksand und Löß, Dünen und Windschliffen an den Randhöhen des Bergischen Landes östlich von Köln. - Neues Jb. Geol. u. Paläont., Abh., 104: 226-254, 10 Abb., 1 Tab., 3 Beil.; Stuttgart.

MaArleveld, G. C. (1976): Periglacial phenomena and the mean annual temperature during the last glacial time in the Netherlands. - Biul. Peryglacjalny, 26: 57-78, 10 Fig.; Lodz.

MEYER, H. H. (1981): Zur klimastratigraphischen und morphogenetischen Auswertbarkeit von Flugdecksandprofilen im norddeutschen Altmoränengebiet - erläutert an Beispielen aus der Kellenberg-Endmoräne (Landkreis Diepholz). - Beiträge zur Glazialmorphologie und zum periglaziären Formenschatz, Bochumer Geogr. Arb., 40: 21-30, 3 Abb., 1 Tab., 5 Fot.; Bochum.

MÜLLER, E. H. (1954): Über die Herkunft des Lösses im Rheinland und im südlichen Westfalen. - Geol. Jb., 69: 401-406, 1 Taf.; Hannover.
Müller, E. H. (1959): Art und Herkunft der Lösse und Bodenbildungen in den äolischen Ablagerungen Nordrhein-Westfalens unter Berücksichtigung der Nachbargebiete. - Fortschr. Geol. Rhld. u. Westf., 4: 255-265, 1 Abb.; Krefeld.

PAAS, W. (1968): Gliederung und Altersstellung der Lösse am Niederrhein. - Fortschr. Geol. Rhld. u. Westf., 16: 185-196, 2 Abb.; Krefeld.

- (1985): Bodenkarte Nordrhein-Westfalen 1:50.000, L 4302 Kleve; Krefeld (Geol. L. A. Nordrhein-Westfalen).

Pons, L. (1957): Die Geologie de Bodemvorning en de waterstaatkundige Ontwikkeling van het Land van Maas en Waal en een gedeelte van het Rijk von Nijmegen. Meded. Sticht. v. Bodemkart., bodemk. Studies, 3: 1-156, 11 Beil.; Wageningen.

Semmel, A. (1990): Periglaziale Formen und Sedimente, 250-260, 5 Abb. - Eiszeitforschung; Darmstadt (Wiss. Buchges.).

SieberTZ, H. (1983): Neue sedimentologische Untersuchungsergebnisse von weichselzeitlichen äolischen Decksedimenten auf dem Niederrheinischen Höhenzug. Beiträge zum Quartär der nördlichen Rheinlande, Arb. Rhein. Landeskde, 51: 51-97, 8 Abb., 6 Tab., 1 Kt.; Bonn.

- (1987): Die stratigraphische und paläogeographische Bedeutung der Steinsohle in den pleistozänen Sedimenten des Niederrheins und angrenzender Gebiete. Decheniana, 140: 193-203, 8 Abb., 2 Tab.; Bonn.

- (1988): Die Beziehung der äolischen Decksedimente in Nordwestdeutschland zur nördlichen Lößgrenze. Eiszeitalter u. Gegenwart, 38: 106-114, 8 Abb.; Hannover.

- (1990): Die Abgrenzung von äolischen Decksedimenten auf dem Niederrheinischen Höhenzug mit Hilfe von Korngruppenkombinationen. - Decheniana, 143: 476-485, 5 Abb., 5 Tab.; Bonn.

- (1992): Konvergenzen sedimentologischer und pedologischer Kartierungsergebnisse auf dem Niederrheinischen Höhenzug in bezug auf ihre paläogeographische Ausdeutung. - Arb. Rhein. Landeskde, 61: Bonn (im Druck).

VIERHUFF, H. (1967): Untersuchungen zur Stratigraphie und Genese der Sandlößvorkommen in Niedersachsen. - Mitt. Geol. Inst. TH, 5: 1-99, 35 Abb.; Hannover.

WiLCKENS, O. (1924): Altalluviale Flugsandbildungen am Niederrhein. - Geol. Rdschau, 15: 289-300; Berlin.

ZaGwijn, W. H. \& Paepe, R. (1968): Die Stratigraphie der weichselzeitlichen Ablagerungen der Niederlande und Belgien. - Eiszeitalter u. Gegenwart, 19: 129-146, 6 Abb.; Öhringen. 